
QL

371

$D 39$

1888

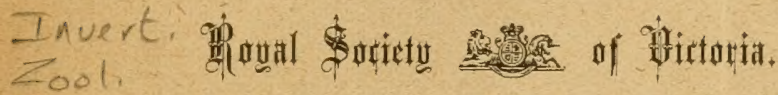

AN ALPHABETICAL LIST OF THE GENERA AND SPECIES OF SPONGES DESCRIBED BY H. J. CARTER, ESQ, F.R.S., TUGETHER WITH A NUMBER OF HIS MORE IMPORTANT REFERENCES TO THOSE OF OTHER AUTHORS, WITH AN INTRODUCTORY NOTICE.

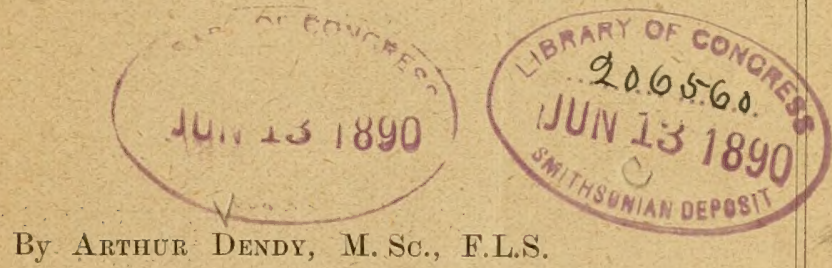

Demonstrator and Assistant Lecturer in Biology in the University of Melbourne. 

AN ALPHABETICAL LIST OF THE GENERA AND SPECIES OF SPONGES DESCRIBED BY H. J. CARTER, Esq., F.R.S., TOGETHER WITH A NUMBER OF HIS MORE IMPORTANT REFERENCES TO THOSE OF OTHER AUTHORS, WITH AN INTRODUCTORY NOTICE.

\author{
By ArThur Dendy, M. Sc., F.L.S.
}

Demonstrator and Assistant Lecturer in Biology in the University of Melbourne.

[Read June 14, 1888].

\title{
I.-Intronuctory Notice.
}

Mr. Carter is, and has for a long time, been justly recognised as the oldest and most experienced of all those who have made a special study of the sub-kingdom Porifera, or Sponges.

He produced his first paper on the subject, entitled "Notes on the Species, Structure, and Animality of the Freshwater Sponges in the Tanks of Bombay," whilst stationed at Bombay as a surgeon in the army. This paper was published in the "Transactions of the Bombay Medical and Physical Society" of $18+7$ (No. 8), and was reprinted in the "Annals and Magazine of Natural History (Series 2, vol. I) in the following year. The last paper on the subject which appeared from his pen, on a collection of sponges from the Mergui Archipelago, was published only last year in the Journal of the Linneun Society of London, and in the same year he published four other papers in the "Annals and Magazine of Natural History."

In the forty-one years from 1847 to 1887 (inclusive), Mr. Carter has published no less than 12.5 papers on sponges, a far greater number than any other author. These papers treat of nearly 800 distinct species of sponges, arranged under over 200 distinct generic names. By far the greater number of these 800 species are described by $\mathrm{Mr}$. Carter for the first time.

Although both his first and last papers were published elsewhere, it was a very rare exception for Mr. Carter to publish anywhere except in the "Annals and Magazine of 
Natural History," which renders the study of his papers, and the production of such a list as the present, a far easier task than it would otherwise have been.

It is much to be regretted, however, that $\mathrm{Mr}$. Carter has never been able to collect his numerous observations and bring them together in a more coherent form. As it is, they remain seattered about in a state of almost hopeless confusion through the 125 papers above mentioned, and without the assistance of some such list as the present, it is difficult to gain a correct idea of his work.

The greater portion by far of Mr. Carter's work is purely systematic, and consists in the description of new species; but he has also written on the Anatomy and Embryology of the group. He commenced, as we liave seen, in 1847, with the study of the Freshwater Sponges, and in the series of papers which he published on this subject, there are some extremely important observations which have bardly attracted as much attention as they deserve. This might be said of a good many of Mr. Carter's observations, and it is possibly due to the fact that they are mostly more or less buried in a mass of purely systematic description, which none but a specialist would ever think of reading. Mr. Carter is a wonderfully careful and accurate observer, and considering the early date at which his observations on Spongilla were made, when the study of sponges was practically virgin soil, his results are most remarkable.

Between the years 1849 and 1868 only two papers appeared in his name, but from 1868 onwards, until the middle of 1887 , not a year has passed in which he has not published something about sponges. The year 1874 was marked by special activity, for in this year he commenced the publication of his "Descriptions and Figures of Deep Sea Sponges and their Spicules from the Atlantic Ocean, dredged up on board H.M.S. Porcupine,"* and in the same year he published two important papers on the Development of Marine Spongest The Porcupine reports are decidedly the most valuable of $\mathrm{Mr}$. Carter's systematic papers, and the papers on the Development of Marine Sponges are undoubtedly the most important of his more purely biological works.

The progress of the work on the Porcupine sponges was interrupted by the publication, in 1875 , of the "Notes

${ }^{*}$ A. M. N. H. Ser, 4. Vol. xiv, p. 207, et seq.

t A. M. N. H. Ser. 4. Vol. xiv, pp. $321,389$. 
Introductory to the Study and Classification of the Spongida." The publication of these notes appears to have been suggested to Mr. Carter by the obvious necessity of having some definite system upon which to arrange his numerous species. The plan of the "Notes" was a comprehensive one. They were to consist of three parts, and were to form a kind of general guide to the whole subject, which was rapidly becoming more and more complex. The three parts were to be-(1) Anatomy and Physiology; (2) Proposed Classification of the Spongida; (3) A further Division into Sub-families, Genera and Species, so far as our knowledge extends.

The first part was a general account of the anatomy and physiology of sponges, including those structures, viz., the skeletal elements, upon which the subsequent classification was based. The second part contains Mr. Carter's scheme of classification, which is sufficiently well known to all spongologists, but which has never obtained general acceptance. He himself, however, has adhered to it systematically, and its appearance at this stage was therefore of great importance to the student of his papers. The promised third part, which should have been a kind of coping stone to the whole structure, most unfortunately never appeared. After the publication of the second part, Mr. Carter returned to his work on the Porcupine Sponges, the descriptions of which he completed in 1876 .

Between 1876 and 1885 there appeared a long series of miscellaneous papers on sponges, which need not here be specially noticed. In 1885 , however, an event took place of special interest to Victorian Naturalists, namely, the appearance of Mr. Carter's first papert on the splendid series of sponges from the neighbourhood of Port Phillip Heads and Western Port, collected and sent to England by Mr. J. Bracebridge Wilson, M.A. Henceforth, Mr. Carter devoted almost all his energy to working out and describing these sponges, and he published a series of fifteen papers upon them in the Annals and Magazine of Natural History. In these fifteen papers he records 211 species and varieties, nearly all of which were new to science and all collected by Mr. Bracebridge Wilson.

After the conclusion of this laborious work, in $1887, \mathrm{Mr}$. Carter published two noteworthy papers of a more strictly

* A. M. N. H. Ser. 4. Vol. xvi, pp. 1, 126.

t A. M. N. H. Ser. 5. Vol. xv, p. 107. 
biological character-the first "On the Position of the Ampullaceous Sac and the Function of the Water CanalSystem in the Spongida ;" and the second, "On the Reproductive Elements of the Spongida." + The latter was his last sponge paper in the Annals and Magazine of Natural History, in which he had now been publishing for 41 years. It was succeeded by his report on the sponges of the Mergui Archipelago, in the Journal of the Linnean Society of London, ${ }_{+}^{+}$which, however, Mr. Carter informs me was written so far back as 1883 .

As Mr. Carter has at length been obliged by the state of his health to cease working at his favourite subject, and as we shall probably have no more papers on sponges from this indefatigable and able naturalist, it has occurred to me that I might perhaps benefit other naturalists as well as myself by the publication of a complete list of all the genera and species which he has described. These are arranged in alphabetical order, with full references to the places where the descriptions are to be found, and it is hoper that the list will serve as an index to Mr. Carter's papers.

The list of genera and species was compiled whilst I was working in the Natural History Department of the British Museum, and it contains 985 references, every one of which was obtained from the original paper. In addition to the species described for the first time by Mr. Carter himself, which are distinguished by the presence of his name attached to them, the list contains a number of his more important references to the species of other authors. Care has been taken to give all the names exactly in the form in which they appear in Mr. Carter's works, and although subsequent research will probably necessitate considerable modification in Mr. Carter's nomenclature, I have felt that it would be out of place to attempt any such modification here.

It may be of especial interest to Victorian Naturalists to observe, that more than a quarter of the species and varieties mentioned in the list are recorded by Mr. Carter from the neighbourhood of Port Phillip Heads and Western Port.

* A. M. N. H. Ser. 5. Vol. xix, p. 203.

† A. M. N. H. Ser. 5. Vol. xix, p. 350.

†Jour. Linn. Soc. Vol. xxi, p. 61. 


\section{II.-Alphabetical List.}

Acanthella cactiformis

$$
\text { , hirciniopsis }
$$

" stipitata

Acanthellina parviconulata

$$
\text { rugolineata }
$$

Acarnus innominatus

Acervochalina claviformis

Agelas dispar

Alcyoncellum corbicula

," speciosum

Aleyonium purpureum

Alectona Higgini

$$
\text { Millari }
$$

Amorphina anonyma

" cancellosa

" megalorhaphis

" nigrocutis

,$\quad$ stellifera

Amorphinopsis excavans

Aphrocallistes beatrix

$$
\text { , Bocagei }
$$

A phroceras asconoides

Aplysina aërophoba

$\begin{array}{ll}, & \begin{array}{l}\text { caspitosa } \\ \text { capensis }\end{array} \\ , & \begin{array}{l}\text { carnosa } \\ \text { cauliformis }\end{array} \\ , & \text { chalinoides } \\ " & \text { compacta } \\ , & \text { compressa } \\ , & \text { corneostellata } \\ & \text { cruor } \\ , & \begin{array}{l}\text { fenestrata } \\ \text { fusca }\end{array}\end{array}$

Carter A.M.N.H. 5, xv, p. 114

A.M.N.H. 5, xvi, p. 364

A.M.N.H. 5, xvi, p. 364

A.M.N.H. 5, vii, p. 380

A.M.N.H. 5, xvi, p. 365

A.M.N.H. 5, xvi, p. 365

A.M.N.H. 4, vii, p. 273

A.M.N.H. 4, xvi, p. 195

Carter A.M.N.H. 5, xviii, p. 376

A.M.N.H. 4, xvi, p. 195

A.M.N.H. $\quad 4, \quad$ xii, p. 367

A.M.N.H. $\quad 4, \quad$ xii, p. 367

A.M.N.H. 4, xvi, p. 197

A.M.N.H. 5, ix, p. 352

Carter A.M.N.H. 5, vi, p. 58 J.R. Micro. Soc., vol. ii, p. 494

, A.M.N.H. 5, xvii, p. 49

, A.M.N.H. 5, xvii, p. 50

" A.M.N.H. 5, vii, p. 368

" A.M.N.H. 5, xvii, p. 50

$"$ A.M.N.H. 5, iii, p. 344

„Jour. Linn. Soc., vol. xxi, p. 77

A.M.N.H. 4, xii, p. 359

A.M.N.H. 4, xii, p. 451

A.M.N.H. $\quad 4, \quad$ xii, p. 359

A.M.N.H. 4, xii, p. 450

A.M.N.H. $\quad 4, \quad$ xvi, p. 199

Carter A.M.N.H. 5, x viii, p. 134

,, A.M.N.H. 5, xviii, p. 135

A.M.N.H. $\quad 4, \quad$ xvi, p. 191

A.M.N.H. 5, ix, p. 270

Carter A.M.N.H. 5, xviii, p. 282

A.M.N.H. 4, xvi, p. 192

A.M.N.H. 5, viii, p. 110

A.M.N.H. $4, \quad$ xvi, p. 191

Carter A.M.N.H. 5, ix, p. 270

P. Acad. Nat. Sci., Philad., 1884, p. 202

Carter A.M.N.H. 4, xvi, p. 192

$\begin{array}{lll}, & \text { A.M.N.H. } & 5, \quad \text { viii, p. } 109 \\ , & \text { A.M.N.H. } & 5, \quad \text { ix, p. } 270\end{array}$

" A.M.N.H. 4, x, p. 105

A.M.N.H. 4, xvi, p. 191

,, A.M.N.H. 5, xviii, p. 286

A.M.N.H. $\quad 5, \quad$ ix, p. 272

Carter A.M.N.H. 5, vi, p. 36 
Aplysina incrustans

" purpurea

$\begin{array}{ll}, & \text { inflata } \\ , & \text { lævis } \\ , & \text { longissuma } \\ , & \text { massa } \\ , & \text { nævus }\end{array}$

Arabescula parasitica Ascaltis Lamarckii Ascetta clathrus Askonema setubalense

Aulodictyon Woodwardii

Axinella atropurpurea chalinoides

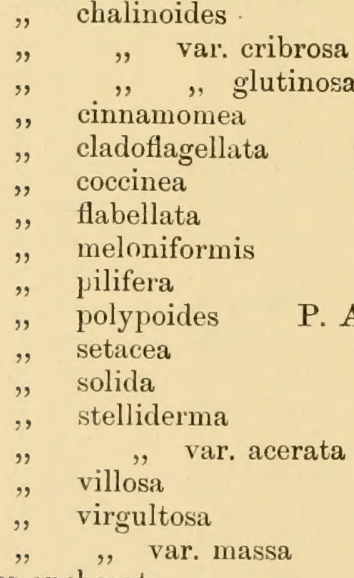

Axos anchorata $\begin{array}{ll}\text {, } & \text { Cliftonii } \\ \text {, fibulata }\end{array}$

"tlabelliformis

", spinipoculum

Azorica Pfeifferæ
Carter

",

,

,

Carter

,

"

,

"

A.M.N.H. 5, viii, p. 107

Carter A.M.N.H. 4, xviii, p. 231 A.M.N.H. 5, viii, p. 108 A.M.N.H. 5, $\quad \mathrm{xv}$, p. 204 A.M.N.H. 5, ix, p. 271 A.M.N.H. 5, xviii, p. 284 A.M.N.H. $\quad 4, x v i i i$, p. 229 A.M.N.H. 5, xviii, p. 285 A.M.N.H. 5, vi, p. 36 A.M.N.H. 5, viii, p. 103 A.M.N.H. 4, xii, p. 464 P. Acad. Nat. Sci., Philad., 1844, p. 208 A.M.N.H. 5, xiv, p. 17 A.M.N.H. $\quad 4, \quad$ xii, p. 361 A.M.N.H. $\quad 4, \quad$ xii, p. 368 A.M.N.H. $\quad 4, \quad$ xii, p. 360 A.M.N.H. $4, \quad$ xii, p. 452 A.M.N.H. $\quad 5, \quad x v i$, p. 359 A.M.N.H. $5, \quad x v i$, p. 358 A.M.N.H. 5, xviii, p. 377 A.M.N.H. $5, \quad$ xvi, p. 359 A.M.N.H. $\quad 4, \quad \mathrm{xvi}$, p. 196 A.M.N.H. 5, xviii, p. $37 i$ A.M.N.H. 5, xviii, p. 378 A.M.N.H. $5, \quad x v i$, p. 361 A.M.N.H. 5, xvi, p. 362 A.M.N.H. 5, xvi, p. 362 P. Acad. Nat. Sci., Philad., 1884, p. 205 Carter A.M.N.H. 5, xvi, p. 359 " A.M.N.H. 5, xvi, p. 362 " A.M.N.H. 5, xvi, p. 360 , A.M.N.H. 5, xvi, p. 360 " A.M.N.H. 5, xvi, p. 361 " Jour. Linn. Soc., vol. xxi, p. 68 ", Jour. Linn. Soc., vol. xxi, p. 68 " A.M.M.H. 5, vii, p. 382 A.M.N.H. 5, ix, p. 288 A.M.N.H. $\quad 4, \quad$ xvi, p. 198 Carter A.M.N.H. 5, vii, p. 383 A.M.N.H. 5, ix, p. 288 A.M.N.H. 5, iii, p. 285 A.M.N.H. 5, iii, p. 286 A.M.N.H. $\quad 4, \quad$ xii, p. 439 A.M.N.H. $\quad 4, \quad$ xii, p. 442 A.M.N.H. 4, xviii, p. 466

Battersbya Bucklandi
A.M.N.H. 4. xvi, p. 199 
Cacospongia cavernosia

, sp.

sp.

Carteriospongia caliciformis Carter

Caulospongia plicata verticillaris

Cavochalina bilannellata , digitata var. arenosa Carter

Cellulophana pileat:

Clualina compressa

, digitata var. arenosa

, inornata

, oculata

, , var. fibrosa

", , repens

" palmata

, polychotoma

, ", var. anchorata

, , , angulita

, ", compressa

", , , moniliformis

", , , oculata

, , , ",, robusta

, , ", trichotoma

, rubens

, seriata

, spinifera

Chalinopsis clathrodes

Chondrilla australiensis

$\begin{array}{ll}, & \text { distincta } \\ , & \text { embolopora } \\ , & \text { mixta } \\ , & \text { nucula }\end{array}$

, papillata

" pliyllodes

, sacciformis

, secunda

Chondrocladia virgata

Chondropsis arenitera

Chondrosia plebeja

reniformis
A.M.N.H. 4, xvi, p. 193

Jour. Limn. Soc., vol. xxi, p. $6 t$

A.M.N.H. 4, vii, p. 276

A.M.N.H. 5, xv, p. 221

A.M.N.H. $4, \quad x v i$, p. 196

A.M.N.H. 4, xvi, p. 196

A.M.N.H. 5, xvi, p. 287

A.M.N.H. 5, ix, p. 281

A.M.N.H. 5, viii, p. 258

A.M.N.H. 5, x, p. 112

Carter A.ML.N.H. 5, ix, p. 280

Midland Naturalist, vol. 3, p. 194

A.M.N.H. 4, xvi, p. 193

P. Acad. Nat. Sci., Philad., 1584, 1. 204

Carter Jour.Lim. Soc., vol.xxi, p. 66

, A.M.N.H. 5, xviii, p. 375

A.M.N.H. 5, x, p. 109

A.M.N.H. 5, xvi, p. 284

Carter A.M.N.H. 5, xvi, p. 289

A.M.N.H. j, xvi, 1. 28.5

A.M.N.H. 5, xvi, p. 284

A.M.N.H. ז, xvi, p. 23.)

A.M.N.H. 5, xvi, p. 284

Carter A.M.N.H. 5, xvi, p. 285

, A.M.N.H. 5, xv, p. 11.

A.M.N.H. 5, xvi, p. 284

A.M.N.H. 5, ix, p. 276

A.M.N.H. 4, xvi, p. 196

Carter Joui. Limn. Soc., vol. xxi, p. $66^{\circ}$

A.M.N.H. 4, xvi, p. 195

Carter A.M.N.H. t, xii, p. 23

A.M.N.H. 5, viii, p. 249

A.M.N.H. 5, viii, ए. 249

A.M.N.H. 5, viii, 1. 249

A.M.N.H. 5, viii, 1. 249

A.M.N.H. 4, xvi, p. 191

A.M.N.H. 5, viii, p. 249

A.M.N.H. 5, ix, p. 268

A.MI.N.H. 5, xviii, p. 277

A.M.N.H. 5, xviii, p. 278

A.M.N.H. 5, viii, 1. 249

Carter A.M.N.H. 5, iii, p. 299

A.M.N.H. 5, xviii, p. 277

A.M.N.H. t, xiv, p. 217

Carter A.M.N.H. 5, xvii, p. 122

A.M.N.H. 5, viii, p. 248

A.M.N.H. 5, viii, 1). 248 
Chondrosia spurea

Carter A.M.N.H. 5, xix, p. 286 tuberculata

A.M.N.H. 5, viii, p. 248

Ciocalyltapenicillus var.aciculata Carter A.M.N.H. 5, xvi, p. 366

'Tyleri

Cladorhiza abyssicola

, var. corticocar

Clathria compressa

, oroides

Clatlirina cavata

, clathrus

, laminoclathrata

, latitubulati

,, osculum

, primordialis

, sulphurei

, tripodifera

Cliona abyssorum , var. gravida ventricosit

, bacillifera

,, caribbica

, celatil

, corallinoides

, ensifer'it

, gracilis

, Howsei

, lobatis

", mazatlanensis

, mucrouata

, notthumbrica

,, subulata

, vastifica

, vermifera

,Wareni

Colummitis squamat:

Cometella pvoula

, simplex

Corallistes aculeata

.. horealis

, Bowerbankii

" clavatella
A.M.N.H. 5, xvi, p. 366

A.M.N.H. 4, xiv, p. 218

A.M.N.H. 4, xviii, p. 319

A.M.N.H. 4, xvi, p. 195

A.M.N.H. 4, xvi, p. 195

Carter A.M.N.H. 5, xvii, p. 502

A.M.N.H. 5, xiv, p. 17

Carter A.M.N.H. 5, xvii, p. 509

A.M.N.H. 5, xvii, p. 515

A.M.N.H. 5, xvii, p. 503

A.M.N.H. 5, xvii, 1. 510

A.M.N.H. 5, xiv, p. 18

Carter A.M.N.H. 5, xvii, P. 505

A.M.N.H. 5, xvii, p. 507

A.M.N.H. 5, xvii, p. 512

A.M.N.H. 4, xiv, p. 249

A.M.N.H. 5, ix, p. 353

Carter Jour. Limn. Soc., vol. xxi, p. 76

A.M.N.H. 5, ix, 1). 346

A.M.N.H. 4, xvi, p. 197

A.M.N.H. 5, ix, p. 349

A.M.N.H. 5, ix, p. 353

A.M.N.H. 5, xviii; p. 458

A.M.N.H. 4, xvi, p. 198

A.M.N.II. 5, ix, p. 353

A.M.N.H. 5, ix, p. 354

A.M.N.H. 5, ix, p. 353

A.M.N.H. 5, ix, p. 353

A.M.N.H. 5, ix, p. 353

A.M.N.H. 5. ix, p. 353

A.M.N.H. 5, ix, p. 354

A.M.N.H. 5, ix, p. 353

A.M.N.H. 5, ix, p. 354

A.M.N.H. 5, ix, p. 353

A.M.N.H. 5, ix, p. 353

Carter A.M.N.H. 5, vii, p. 370

A.M.N.H. 5, viii, p. 252

Carter A.M.N.H. 4, xviii, p. 388

A.M.N.H. 4, xviii, p. 395

A.M.N.H. 5, vi, p. 143

, A.M.N.H. 4, xii, p. 439

A.M.N.H. $4, \quad$ xii, p. 443

A.M.N.H. 4, xviii, p. 460

A.M.N.H. 4, xii, p. 438 
Corallistes elegantior

" elegantissima

" microtuberculatus

" noli tangere

", polydiscus

, typus

, verrucosia

Cornulum textile

Corticium abyssi

$\begin{array}{ll}, & \text { australiensis } \\ , & \text { candelabrum } \\ , & \text { Kittonii } \\ & \\ " & \text { parasiticum } \\ " & \text { plicatum } \\ , & \text { stelligerum } \\ . & \text { versatile } \\ , & \text { Wallichii }\end{array}$

Coscinoderma lanuginosum

Crateromorpha Meyeri

Cribrella hospitalis

Dactylia chaliniformis , impar

, palmata

Dactylocalyx Bowerbankii

$\begin{array}{ll}., & \text { Masoni } \\ , & \text { polydiscus } \\ . \quad & \text { Prattii } \\ , \quad \text { pumicea } \\ " \quad \text { pumiceus } \\ , \quad \text { subglobosa }\end{array}$

Dactylocalycites callodiscus Carter ellipticus

Carter

$"$

..

Carter

Curter

C'arter

.


Dactylocalycites Vicaryi Carter

Darwinella australiensis

Dendrilla rosea var: digitata "

Dercites haldonensis

Dercitus niger

Desmacella pumilio

Desmacidon ægagropila

" fistulosa

", var. tuliginosa Carter

.. Jeftreysii

titubans

Dictyocylindrus abyssi

$\begin{array}{ll}" & \text { abyssorum } \\ " & \text { aceratus } \\ " & \text { anchorata } \\ " & \text { cacticutis } \\ \text { ". } & \text { dentatus } \\ \text { lispidus }\end{array}$

Discodermia ispera

laciniatus manatrensis , piniformis ", pinnatificlus Pykii ramosus reticulatus sessilis simplex

Vickersii virgultosus

$" \quad$ levidiseus

., papillata

", polydiscus

.. seeptrelliferia

". sinuosa

", spinispirulifera

Donatia an'untium

.. lyncurium

, multitida

Dotona pulchella

Dysidea antiqua

" chaliniformis

", fragilis
A.M.N.H. 4, vii, p. 123

A.M.N.H. $\quad 5, \quad \mathrm{xv}, \mathrm{p} .202$

A.M.N.H. 5, xviii, p. 281

A.M.N.H. $\quad$ 4, vii, p. 130

A.M.N.H. 4, vii, $p . \quad 3$

A.M.N.H. 4, xvi, 1. 1!9

A.M.N.H. t, xiv, p. 250

A.M.N.H. 4, xvi, p. 197

A.M.N.H. 5, x, p. 121

A.M.N.H. 5, x, p. 121

A.M.N.H. 5, vi, p. 37

A.M.N.H. 5, x, p. 121

A.M.N.H. 4, xvi, p. 197

Carter A.M.N.H. 4, xii, p. 29

A.M.N.H. 4, xviii, p. 232

"Jour. Linn. Soc., vol. xxi, p. 67

A.M.N.H. 4, xiv, p. 251

A.M.N.H. 5, xvi, p. 354

A.M.N.H. 4, xvi, p. 198

A.M.N.H. 4, xvi, p. 196

Jour. Linn. Soc., vol. xxi, p. 66

Carter A.M.N.H. 5, iii, p. 296

A.M.N.H. 5, vi, 1. 37

A.M.N.H. 5, xvi, p. 354

A.M.N.H. 5, xvi, p. 353

A.M.N.H. 5, iii, p. 297

A.M.N.H. 4, xvi, p. 195

A.M.N.H. 5, vii, p. 377

A.M.N.H. 5, vi, p. 38

A.M.N.H. 4, xviii, p. 234

A.M.N.H. 5, iii, p. 292

A.M.N.H. 4, xviii, p. 234

Garter A.M.N.H. 5, vi, p. 147

A.MI.N.H. 5, vi. p. 149

A.M.N.H. 5, vi, p. 146

A.M.N.H. 4, xviii, p. 462

Carter A.M.N.H. 5, vii, p. 372

A.M.N.H. 5, vii, p. 372

A.M.N.H. 5, vi, p. 148

A.M.N.H. 4, xvi, p. 198

A.M.N.H 5, ix, p. 358

Jour. Limm. Soc., vol, xxi, p. 77

Carter A.M.N.H. 5, ix, p. 358

$\begin{array}{llll} & \text { A.M.N.H. } & 5, & \text { vi, p. } 57 \\ & \text { A.M.N.H. } & 5, & \text { i, p. } 139\end{array}$

" A.M.N.H. 5, xv, p. 217

A.M.N.H. t, xvi, p. 193 
Dysidea hirciniformis

, Kirkii

, ramoglomerata

", , var. granulata

", ", ramotubulata

", tenerrima Carter P.Acad.Nat.Sci., Philad., 1884, p. 203

, tubulosa

Echinoclathria favus

\section{"}

var, aren gracilis

nodosa

"

$"$

subhispida

tenuis

Echinonema anchoratum

,

crespitosa

flabelliformis

incrustans

pectiniformis

typicum

vasiplicata

Ecionemia compressa

nana

Ectyon cylindricus

"flabelliformis

," mauritianus

" sparsus

Ectyonopsis ramosa

Esperia borassus

, Cunninghami

" cupressiformis

", , , hamatifera

, indica

,, lævis

, obscura

" parasitica

, placoides

, plumosa
A.M.N.H. 4, xviii, p. 232

A.M.N.H. 5, xv, p. 215

Carter A.M.N.H. 5, xv, 1, 21

A.M.N.H. 5, vii, p. 337

A.M.N.H. 5, xr, p. 216 Carter Jour. Linn. Soc., vol. xxi, p. 64

Carter A.M.N.H. 5, ix, p. 275

A.M.N.H. 5, xvi, p. 292

A.M.N.H. 5, xvi, p. 350

A.M.N.H. 5, xvi, p. 356

A.M.N.H. 5, xvi, p. 356

A.M.N.H. 5, xvi, p. 356

A.M.N.H. 5, xvi, p. 355

A.M.N.H. 5, vii, p. 379

A.M.N.H, 5, xvi, p. 352

A.M.N.H. 5, xvi, p. 352

A.M.N.H. 5, xvi, p. 353

A.MI.N.H. 5, xvi, p. 353

A.M.N.H. 4, xvi, p. 195

A.M.N.H. 5, vii, 1, 37.

A.M.N.H. 5, $\quad \mathrm{x}, \mathrm{p} .114$

A.M.N.H. 5, xi, p. 362

Carter A.M.N.H. 5, xi, p. 362

$\begin{array}{lll}\text { A.M.N.H. } & 5, & \text { xii, p. } 314 \\ \text { A.M.N.H. } & 5, & \text { xii, p. } 311\end{array}$

" A.M.N.H. $\quad 5, \quad$ xii, p. 310

A.M.N.H. 4, vii, p. 270

A.M.N.H. 4, xvi, p. 195

A.M.N.H. 5, ix, p. 281

Carter A.M.N.H. 5, xii, p. 315

A.M.N.H. 4, xviii, p. 317

"A.M.N.H. 5, ix, p. 300

A.M.N.H. 4, xiv, p. 215

A.M.N.H. 4, xviii, p. 318

A.M.N.H. 4, xviii, p. 471

Jour. Linn. Soc., vol. xxi, p. 72

A.M.N.H. $5, \quad \mathrm{ix}, \mathrm{p} .291$

A.M.N.H. 5, ix, p. 299

A.M.N.H. 5, xv, p. 108

A.M.N.H. 5, xviii, $\mathrm{p} .455$

Carter A.M.N.H. 4, xviii, p. 316

A.M.N.H. 5, ix, p. 299 Jour. Linn. Soc, vol. xxi, p. 72 
Esperia serratohamata

,. socialis

"tunicata

, villosa

Esperiopsis villosa

Esperites gigantens

, haldonensis

Euplectella aspergillum

.. $\quad$ encumer

Eurete farreopsis

Euspongia anfractuosa

$$
\begin{aligned}
& \text { " compacta } \\
& \text {.. infundibuliformis }
\end{aligned}
$$

Farrea densa

„, facunda

", fecunda

" infundibularis

" infundibuliformis

„ocea

Fibularia massa

1:ilinsa

Fibulia carnosa

Forcepia colonensis

.. crassanchorata

Geelongia rasiformis

Geodia arabica

., itreolita

.. canaliculata

., gibberosa

., globostellifera

, megastrella

" , var. levispina

", nodastrellat
Carter A.M.N.H. 5, vi, p. 49 A.M.N.H. t, vii, p. 276 A.M.N.H. 5, vi. p. 49

Carter A.M.N.H. t, xiv, p. 213 A.M.N.H. 5. ix, p. 296 " A.M.N.H. 4, vii, p. 131 A.M.N.H. 4, xviii, p. 459 (arter A.M.N.H. 4, vii, p. 1:31 A.M.N.H. 4, xii, 1. 35!! A.M.N.H. 4, xii, p. 3606 A.M.N.I. 4, xvi, p. 199 A.M.N.H. 4, xii, p. 362 A.M.N.H. 4, xii, p. 367 A.M.N.H. 4, xvi, p. 200 Carter A.MIN.H. 4, xix, p. 122 A.M.N.H. 5, xv, p. 316 A.M.N.H. 5, x, p. 106 A.M.N.H. 5, xviii, p. 374

A.M.N.H. t, xii, p. 463 A.M.N.H. 4, xii, p. 4.54 A.M.N.H. 4, xii, p. 360 Carter A.M.N.H. t. xii, p. 360 A.M.N.H. 4, xii, p. 448 A.M.N.H. 4, xii, p. 360 A.MI.N.H. $4, \quad$ xii, p. 4.54 A.M.N.H. 5, $\quad \mathrm{xv}, \mathrm{p} .38 \mathrm{~s}$ Carter A.M.N.H. 5, ix, p. 2-2. A.M.N.H. 5, ix, p. 283 Jour. Linn. Soc, rol, xxi, p. $\mathrm{I}$ ('arter A.M.N.H. 5, xvii, p. 51 .M.N.H. 4, xiv, p. 248 A.M.N.H. 5, xv, p. 110 A.M.N.H. 5, xvii, p. $5: 3$ A.M.N.H. 5, xv, p. 111

9

A.M.N.H. $5, \quad x v, p .120$ A.M.N.H. 5, xv, p. 306 A.N.N.H. 4, iv, p. 4 A.M.N.H. 4, xvi, p. 198 A.M.N.H. 5, vi, p. 13 ? I.M.N.H. 5, xi, p. 346 A.M.N.H. 5, ix, p. 364 (arter A.M.N.H. 5, vi, p. 1:3t A.M.N.H. 4, xviii, p. 400 A.M..N.H. $\quad$ t, xviii, p. 401 A.M.N.H. 4, xviii, p. 397 
Geodial perarmat:

, ramodigitatia

, tuberculosa

, tumulosa

, zetlandica

Geodites haldonensis

Gomphites Parfittii

Grantia ciliata

parsiceps , ? var. splinispiculum clathrus

" compressa
A.M.N.H. 5, vi, p. 131
Carter A.M.N.H. 5, vi, p. 133
A.M.N.H. 5, ix, p. 364

P. Acad. Nat. Sci., Philad., 1884, p. 208

A.M.N.H. 5, ix, p. 362

A.M.N.H. 4, xvi, p: 198

Carter A.M.N.H. 4, vii, p. 129

A.M.N.H. 4, vii, p. 127

" A.M.N.H. 4, vii, p. 127

Midland Naturalist, vol. iii, p. 195

Carter A.M.N.H. 5, xiii, p. 156

,

A.M.N.H. 4, xviij, p. 168

A.M.N.H. 5, xiv, 1) 17

Midland Naturalist, vol. iii, p. 195

, var. fistulata Carter A.M.N.H. 5, xviii, p. 3

", subhispicla

, tesselata

(isayellat cyathophoræa

, A.M.N.H. 5, xviii, 1. 36

Midland Naturalist, vol. iii, p. 195

('arter A.M.I.II. t, iv, p. 190

A.M.N.H. 4, xvi, p. 198

A.M.X.H. 5, viii, p. 251

Guitarra fimbriata

Gummina ecaudata

A.MI.N.H. 4, xiv, p. 210

,$\quad$ gliricauda

A.M.N.H. 5, viii, p. 248

A.M.N.H. 5, viii, p. 248

Carter A.M.N.H. 4, xiv, p. 252

Habrodictyon corbicula

Halichondia alyysi

aceratospiculum ,

A.M.N.H. 4, xii, p. 361

A.M.N.H. 4, xii, p. 361

Carter

A.M.N.II. t, xiv, p. 245

A.MIN.H. t, xviii, 1. 315

, $\quad$ axgigropilat

A.M.N.H. 5, vi, p. 49

" $\quad$ birotulata

A.M.N.H. 4, xvi, 1. 197

A.M.N.H. 5, vi, p. 48

A.M.N.H. 5, xvii, p. 52

Jour. Limn. Soc., vol. xxi, p. 72 carnosa Phil. Trans. Fioy. Soc., vol. clxviii, p. 287

A.II.N.H. 5, ix, p. 353

compressi Carter A.M.N.H. 5, xviii, p. 450

ficus A.M.N.H. 4, xvi, p. 197

A.M.N.H. 5, ix, p. 353

foliata

A.M.N.H. 4, xviii, p. 310

A.M.N.H. 4, xiv, p. 246
, $\quad$, val. bulbosa Carter A.M.N.H. 4, xvii, p. 312

59.

Hyndmani

A.M.N.H. 4, xvi, p. 197

incrustans

A.MI.N.H. 4, xvi, p. 197

P. Acad. Nat. Sci., Philad., 1884, P. 206

infrequens Carter A.M.N.H. 5, vii, P. 369 
Halichondria isodictyalis Carter A.M.N.H. 5, ix, p. 285

A.M.N.H. 5, xvii, p. 52

$\begin{array}{ll}\text {, } & \text { maculans } \\ , & \text { palmata } \\ \text { panicea }\end{array}$

A.M.N.H. $\quad 4, \quad x v i$, p. 195

A.M.N.H. 4, xvi, p. 194

A.MI.N.H. $4, \quad$ xvi, p. 196

Pliil. Trans. Roy. Soc., vol, clxviii, p. 286

Midland Naturalist, vol. iii, p. 194

P. Acad. Nat. Sci., Philad., 1884, p. 206

Jour. Limn. Soc., vol. xxi, p. 69

" phlyctenodes Carter A.M.N.H. 4, xviii, p. 314

" plumosa A.M.N.H. 4, xvi, p. 195

" Phil. 'Trans. Roy. Soc., vol. clxviii, p. 287

A.M.N.H. 5, vii, p. 368

$"$ pustulosa Carter A.M.N.H. 5, ix, p. 285

A.M.N.H. 5, xviii, p. 450

" sanguinea A.M.N.H. 4, xvi, p. 197

Phil. 'Trans. Koy. Soc., vol. clxviii, p. 287

P. Acad. Nat. Sci, Philad., 1884, p. 208

$"$ scabida. Carter A.M.N.H. 5, xv, p. 112

A.M.N.H. 5, xviii, p. 449

$"$ seriata A.M.N.H. 4, xvi, p. 196

$"$ simulans A.M.N.H. 4, xiv, p. 331

A.M.N.H. 4, xvi, p. 194

" stelliderma Carter A.M.N.H. 5, xviii, p. 451

", suberea A.M.N.H. 4, xvi, p. 197

"suberia A.M.N.H. 5, ix, p. 353

Halicnemia patera A.M.N.H. 4, xvi, p. 198

Halisarer ascidiarum Carter A.N.N.H. 5, ix, p. 357

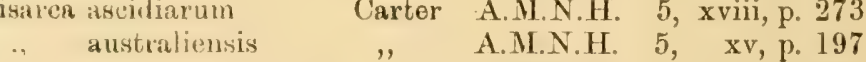

A.M.N.H. 5, xviii, p. 273

" "val, "trenacea ", A.M.N.H. 5, xviii, p. 277

., bassangustiarum " A.M.N.H. 5, vii, p. 373

.. oruenta " A.M.N.H. 4, xviii, p. 228

A.M.N.H. 5, viii, p. 247

,. bujardinii A.M.N.H. 4, xvi, p. 191

A.M.N.H. 5, viii, p. 245

" guttula A.M.N.H. 5, viii, p. 246

$"$ lobularis A.M.N.H. 4, xiii, p. 434

A.II.N.H. 4, xvi, p. 191

A.M.N.H. 5, viii, p. 245

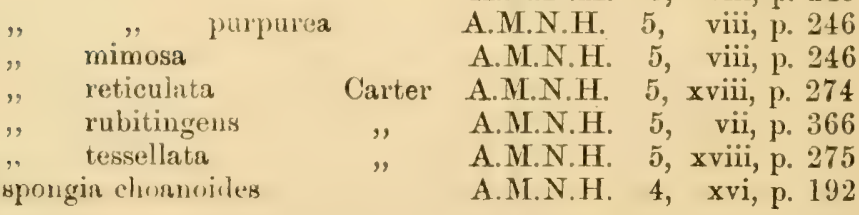


Halispongia Mantelli

$$
\text { ventriculoides }
$$

Hemiasterella affinis

Heteropia compressa

$$
\text { typus }
$$

$\begin{array}{ll}, & \text { erecta } \\ , & \text { macera } \\ , & \text { patulosculifera } \\ . & \text { pluiosculifera } \\ , & \text { polyperistomia } \\ , & \text { spissa }\end{array}$

Hexactinella ventilabrum
A.M.N.H. 4, xvi, p. 193

A.M.N.H. 4, xvi, p. 193

Garter A.M.N.H. 5, iii, p. 147

A.M.N.H. 5, iii, p. 146

A.M.N.H. 5, xviii, p. 51

A.M.N.H. 5, xviii, p. 53

A.M.N.H. 5, xviii, p. 50

A.M.N.H. 5, xviii, p. 49

A.M.N.H. 5, xviii, p. 52

A.M.N.H. 5, xviii, p. 47

A.M.N.H. 5, xviii, p. 54

A.M.N.H. $5, \quad \mathrm{xv}, \mathrm{p} .397$

Higginsia cotalloides P. Acad. Nat. Sei., Philat., 1884, p. 205

" " var. massalis Carter A.M.N.H. 5, xvi, p. 357

A.M.N.H. 5, xvi, p. 293

A.M.N.H. 5, xvi, p. 358

Hircinia ar undinacea natilensis ,

$"$

A.M.N.H. 5, vi, p. 36

" caracasensis

", elathratia

$"$ communis

" flatellopilumatit

, flagelliformis

, fuscar

,, intertextir

, panicea

, pulchra

," rectilinea

, solida

" tubulosa Carter

", typica

" variabilis

A.M.N.H. 5, ix, p. 273

A.M.N.H. 5, vii, p. 366

A.M.N.H. 5, xv, p. 314

A.M.N.H. 5, $\quad x v, p .313$

A.M.N.H. 5, xviii, p. 372

, sp.

A.M.N.H. 5, vi, p. 36

A.MI.N.H. 5, $\mathrm{xv}$, p. 120

A.N.N.H. 5, xv, p. 312

A.M.N.H. 4, xvi, p. 192

Carter A.M.N.H. 5, xv, p. 314

A.M.N.H. 5, xviii, p. 373

Carter A.M.N.H. 5, xv, p. 310

Histodermil appendiculatum Carter A.M.N.H. 4, xiv, p. 220

Histioderma polymasteides " veriucostun

Holasterella conferta Wrightii

Holopsamma crassa

$\begin{array}{ll}" & \text { fuliginosa } \\ " & \text { levis } \\ " & \text { laminefa vosa } \\ " & \text { turbo }\end{array}$

Holtenia Carpenteri

$» \quad$ Pourtalesii
A.M.N.H. 5, xviii, p. 453

A.M.N.H. 5, xviii, p. 452

A.MI.N.H. 5, vi, p. 211

A.H.N.II. 5, iii, l. 141

A.M.N.H. 5, xv, p. 211

A.M.N.H. 5, xv, p. 213

A.M.N.H. $5, \quad \mathrm{xv}, \mathrm{p} .212$

A.M.N.H. 5, xv, p. 212

A.M.N.H. 5, $\quad \mathrm{xv}$, P. 213

A.N.N.H. $\quad t$, xii, p. 362

A.M.N.H. $\quad 4, \quad$ xii, p. 372

A.M.N.H. $\quad 4, \quad$ xvi, p. 199

A.M.N.H. 4, xii, p. 361 


\section{6}

Hyalonema cebuense

Iusitanicum

Sieboldii

A.M.N.H. 4, xii, p. 370

A.M.N.H. 4, xvi, p. 199

A.M.N.H. 4, xii, p. 362

A.M.N.H. 4, xvi, p. 199

A.M.N.H. 4, xii, p. 362

A.M.N.H. $4, \quad$ xvi, p. 199

A.M.N.H. $5, \quad x v, p .387$

A.M.N.H. 5, i, p. 129

Smithii

Hymedesmia capitatostellifera Carter

A.MI.N.H. 5

vi, p. 51

A.M.N.H. 4, xvi, p. 197

Moorei

Carter

A.M.N.H. 5, vi, p. 50

A.M.N.H. 5, vi, p. 51

A.M.N.H. 5, vi, p. 50

A.M.N.H. $5, \quad$ vi, p. 52

A.M.N.H. 5, ix, p. 352

A.M.N.H. 4, xvi, p. 199

A.M.N.H. 5, ix, p. 353

A.M.N.H. 4, vii, p. 276

A.M.N.H. 4, xvi, p. 197

Midland Naturalist, rol. iii, p. 194

plumosit

pulvinatus

sanguinea

suberia

Hymeraphia microcionides

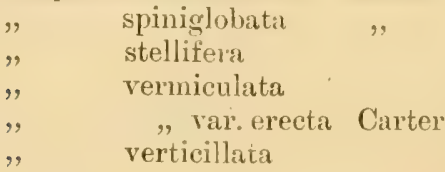

Hymerhaphia clavata

$"$ eruea Carter A.M.N.H. 5, vi, p. 46

$"$ eruea Carter A.M.N.H. 5, vi, p. 46

A.M.N.H. $5, \quad$ ix, p. 350

A.M.N.H. 4, xvi, p. 197

Midland Naturalist, vol, iii, p. 195

Midland Natiuralist, vol. iii, p. 195

('arter A.M.N.H. 4, xviii, p. 390

A.M.N.H. 5, iii, p. 301

A.M.N.H. $\quad 4, \quad$ xvi, p. 195

A.M.N.H. 4, xvi, p. 196

A.M.N.H. 4, xviii, p. 307

A.M.N.H. $4, \quad$ xvi, p. 198

A.M.N.H. 4, xviii, p. 321

A.M.N.H. 5, vi, p. 46

A.M.N.H. 5, vii, 1. 368

unispiculum
, v.M.N.H.

Hypograntia extusarticulata Carter A.M.N.H. 5, xviii, p. 43

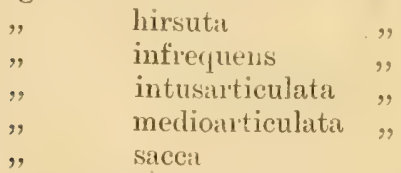

Ianthella (Alabelliformis)

Iphiteon panicea

Isodictya mirabilis

Normani

A.M.N.H. 5, xviii, p. 41

A.M.N.H. 5, xviii, p. 39

A.M.N.H. 5, xviii, p. 45

A.M.N.H. 5, xviii, p. 46

A.M.N.H. 5, xviii, p. 42

A.M.N.H. 4, xvi, p. 191

A.M.N.H. 4, xii, p. 35 T

A.M.N.H. 4, xvi, p. 196

Midland Naturalist, vol. iii, p. 194 
Isodictya rosea

A.M.N.H. 4, xvi, p. 196

Phil. Trans. Roy. Soc., vol. clxviii, p. 286 , simulans var. cancellata

Carter Jour. Linn. Soc, vol, xxi, p. 69 " sub. var. albida

Curter Jour. Linn. Soc., vol. xxi, p. 69 sub. var. fusca

Carter Jour. Linn. Soc, vol. xxi,p. 69

", , , incrustans ", Jour. Linn. Soc., vol. xxi, p. 70

" " " " tubuloramosa ", Jour. Linn. Soc., vol. xxi, p. 70

" spinispiculum $\quad$ A.M.N.H. 4, xviii, p. 310

$"$ varians $"$ A.M.N.H. 4, xvi, p. 196

Kaliapsis cidaris

Labaria hemisphærica

Lacinia stellifica

Latruncula corticata

Latrunculia corticata

$\begin{array}{ll}" & \text { cratera } \\ , \quad & \text { purpurea }\end{array}$

Leiodermatium lynceus

Lelapia australis

ramosum

Leucaltis floridana rar. anstraliensis

Leucetta clathrata

Leuconia compacta

$\begin{array}{ll}\text { echinata } & \text { crinaceus } \\ , & \text { fistulosa var, australiensis }\end{array}$

" Carter.

, Johnstonii

, "var. australiensis

$"$ hispida

, lohata

", multifida

" nivea var. australiensis ,

Leucophlrea massalis

Leucophleus compressus massalis

Leucosolenia contorta
A.M.N.H. 4, xii, p. 438

A.M.N.H. 4, xii, p. 441

A.M.N.H. $\quad 4, \quad$ xi, p. 275

A.M.N.H. t, xii, p. 362

A.M.N.H. 4, xii, p. 373

A.M.N.H. t, xvi, p. 200

A.M.N.H. 5, viii, p. 249

Carter A.M.N.H. 5, iii, p. 298

A.M.N.H. 5, viii, p. 252

A.M.N.H. 5, ix, p. 355

A.M.N.H. 4, xviii, p. 396

Carter A.M.N.H. 5, vii, p. 380

A.M.N.H. $\quad 4, \quad$ xii, p. 439

A.M.N.H. 4, xii, p. 439

A.M.N.H. 5, xriii, p. 138

A.M.N.H. 5, xviii, p. 148

Carter A.M.N.H. 5, xviii, p. 145

A.M.N.H. 5, xi, p. 33

A.M.N.H. 5, xviii, p. 144

A.M.N.H. 5, xviii, p. 129

A.M.N.H. 5, xriii, p. 130

A.M.N.H. 5, xviii, p. 127

A.M.N.H. t, viii, p. 3

A.M.N.H. 5, xviii, p. 133

A.M.N.H. 5, xviii, p. 128

A.MI.N.H. 5, xviii, p. 143

A.M.N.H. 5, xviii, p. 111

A.M.N.H. 5, xviii, p. 131

A.M.N.H. 5, xvi, p. 366

A.M.N.H. 5, xii, p. 324

A.M.N.H. 5, xii, p. 323

Hidland Naturalist, vol. iii, p. 195 
Leucosolenia coriacea

A.M.N.H. 5, xiv, p. 17 " lacunosa var. Hillieri Carter A.M N.H. 5, xiv, p. 24 Lithospongites Kittonii

A.M.N.H. 4, xii, p. 439 Lubomirskia bacillifera

A.M.N.H. 5, vii, p. 103 ", baicalensis

A.M.N.H. 5, vii, p. 103

Luffaria canliformis

papyracea

" , var. elongo-reticulata ,

$", \quad, \quad$ rufa

" digitata

$"$ fistularis

" ramosa

, sessilis

MacAndrewia azorica

Macandrewia azorica

Melonanchora elliptica

Meyenia anonyma

$$
\begin{array}{ll} 
& \text { Baileyi } \\
" & \text { Capewelli } \\
" & \text { fluviatilis }
\end{array}
$$

" " var. augustibirotulata

Carter

, , " gracilis ,

", gregaria oracilis ",

, Leidii

, plumosar

Meyerina claveformis

" claviformis

Microciona acerato-obtusa Carter Jour. Linn. Soc., vol. xxi, p. fi

$\begin{array}{ll}, & \text { affinis } \\ , & \text { armata } \\ , & \text { atrosanguinea } \\ & \\ , & \text { bulboretorta } \\ " & \text { curvispiculifera } \\ " & \text { fascispiculifera } \\ " & \text { intexta } \\ , & \text { jecuseulum } \\ , & \text { longispiculum } \\ " & \text { plana } \\ , & \text { plumosa }\end{array}$

Carter

A.M.N.H. 5, vii, p. 104

A.M.N.H. 5, ix, p. 268

A.M.N.H. 5, ix, p. 269

A.M.N.H. 5, ix, 1. 26?

A.M.N.H. 5, $\quad \mathrm{xv}$, 1. 201

A.MI.N.H. $\quad 4, \quad x v i$, p. 191

A.MI.N.H. 4, xvi, p. 191

A.M.N.H. 4, xvi, p. 191

A.M.N.H. 4, xii, p. 438

A.M.N.H. $4, \quad$ xii, p. 441

A.M.N.H. $\quad 4, \quad x v i, p .199$

A.M.N.H. 4, xviii, p. 464

Carter A.M.N.H. 4, xiv, p. 212

A.MI.N.H. 5, vii, p. 95

A.MI.N.H. 5, vii, p. 95

A.M.N.H. 5, vii, p. 93

A.M.N.H. 5, vii, p. 92

A.M.N.H. 5, $\quad x v$, p. 454

A.M.N.H. 5. xvi, p. 180

A.M.N.H. 5, vii, p. 91

A.M.N.H. 5, vii, p. 91

A.ML.N.H. 5, vii, p. 94

A.M.N.H. 4, xvi, p. 200

A.II.N.II. $4, \quad x, p, 110$

A.M.N.H. $\quad 4, \quad$ xii, p. 362

A.M.N.H. 4, xii, p. 372

A.M.N.H. 5, vi, p. 41

A.M.N.H. $\quad$ t, xiv, p. 457

A.M.N.H. 5, vi, p. 40

A.M.N.H. t, xvi, p. 195

A.M.N.H. .), vi, p. 40

Carter A.M.N.H. 5, vi, p. 41

A.M.N.H. i, vi, p. 43

A.M.N.H. 5, vi, p. 44

A.M.N.H. t, xviii, p. 238

A.M.N.H. 4, xviii, p. 237

Carter A.M.N.H. t, xviii, p. 237

A.M.N.H. 4, xviii, p. 238 Midland Naturalist, vol. iii, p. 194 
Microciona pusilla quadriratiata quinqueraliata

Monanchora clathrata

Monilites haldonensis

Myliusia callocyathes

$$
\text { , Grayi }
$$

Ophiraphidites tortuosus

Usculina polystomella

Pachastrella abyssi

$\begin{array}{ll}, & \text { amygdaloides } \\ , & \text { geodioides } \\ , & \text { intexta } \\ , & \text { parasitica }\end{array}$

Patchymatismat Johnstonia Johnstonii

Paraspongia laxa

Parmula Batesii

, Brownii

Patuloscula procumbens

$$
\text { , val: flabelliformis , }
$$

P'eriphragella Elisse

\section{Cartel:}

A.M.N.H. 4, xviii, p. tis A.M.N.H. 5, viii, 1. 251

Carter
,,
,
,

A.M.N.H. 4, xvi, p. 199 A.M.N.H. 4, xviii, p. 406 A.M.N.H. 4, xviii, p. 407 A.M.N.H. 4, xviii, p. 409 A.M.N.H. 4, xviii, 1\%. 410 A.M.N.H. 5, vi, p. 60 , I.M.N.H. 5, xv, p. t(1:; A.M.N.H. 4, iv, p. is A.M.N.H. 4, xvi, p. 19i Citrter A.MI.N.H. 5, xr, 1. 318 I.M.N.H. 5, vii, p. !!! A.II.N.H. 5, vii, p. y!

Carter A.M.N.H. 5, ix, p. 3(i.) A.M.N.H. 5, xvi, p. 286 A.M.N.H. 5, xvi, p. 286 A.M.N.H. 5, xv, p. 391 Phakellia brassicata

$\begin{array}{ll}, & \text { crassa } \\ , & \text { flabellata } \\ , & \text { infundibuliformis } \\ , & \text { papyracea } \\ , & \text { ramosa } \\ , & \text { ventilabrum }\end{array}$

Carter A.M.N.H. 5, xvi, p. 36;3 , A.M.N.H. 5, xvi, 1. :36:3 , A.M.N.H. 5, xvi, p. $36 ; 3$ A.M.N.H. 4, xviii, p. 2+0 Carter A.M.N.H. 5, xviii, p. 37 ! A.M.N.H. 5, xii, p. 318 A.M.N.H. $\quad t, \quad$ xvi, p. 196 A.M.N.H. 4, xviii, p. 23!) Carter A.M.N.H. 5, xviii, p. 379 A.M.N.H. t, xii, p. 362 A.M.N.H. 4, xii, p. 37. A.M.N.H. 4, xii, p. $36 \%$ A.M.N.H. $4, \quad$ xii, p. 372 Phloeadictyon birotuliferum Carter $\begin{array}{ll}, & \text { cohcrens } \\ , & \text { inondurasensis } \\ & \text { isodictyiforme }\end{array}$ $\begin{array}{ll}" & \text { cohcrens } \\ , & \text { hondurasensis } \\ , & \text { isodictyiforme }\end{array}$ A.M.N.H. 5, xviii, p. 147 A.M.N.H. 5, xviii, p. $4+6$
,9

,

, A.M.N.H. 5, x, p. 12.2
A.M.N.H. $5, \quad$ x, p. 12: 
Phlœodictyon niduliformis Carter

,

,

Phorbiss amaranthus

" anchorata

, tibulata

Phycopsis fruticulos:

,. hirsuta

Placospongia melobesioides

Plumohalichondria arenacea Carter $\begin{array}{lc}, & \text { mammillata } \\ , & \text { microcionides }\end{array}$ Podospongia Lovenii

Polymastia bicolor

Carter

". val. crassa
.
", massalis
,$\quad$ stipitatial

Pseudoceratina crateriformis

, durissima typica

Pseudoesperia enigmatica

Pseudohalichondria clavilobata

Ptilocaulis gracilis

Pulvillus Thomsonii rigiclus

Purisiphonia Clarkei

Racodisculat asteroides

Radiella spinularia

Raphiophora patera

Raphyrus Griftithsii
A.M.N.H. 5, $\quad$ x, p. 123

A.M.N.H. 5, - xii, p. 326

A.M.N.H. 5, x, p. 123

A.M.N.H. j, ix, 1. 285

Carter A.MI.N.H. 5, ix, p. 288

A.M.N.H. 5, ix, p. 288

A.M.N.H. 5, xii, p. 320

A.M.N.H. 5, xii, p. 319

A.M.N.H. t, xvi, p. 198

A.M.N.H. 5. vi, p. .3

A.M.N.H. 5, is, p. 3.5

A.M.N.H. 5, xvi, p. 367

A.M.N.H. 5, xvi, p. 355

A.M.N.H. 4, xviii, p. 236

A.M.N.H. 5, xviii, p. 376

A.M.N.H. t, xviii, p. 396

A.M.N.H. 5, xvii, p. 119

A.M.N.H. 5, xvii, p. 120

A.M.N.H. 5, xvii, p. 119

A.M.N.H. 5, xvii, p. 121

A.M.N.H. 4, xviii, p. 393

A.M.N.H. 5, xv, p. 205

A.M.N.H. 5, xv, p. 204

A.M.N.H. 5, xviii, p. 287

A.M.N.H. 5, xviii, p. 455

A.M.N.H. 5, xviii, p. 454

A.M.N.H. 5, xii, p. 321

A.M.N.H. 5, xii, p. 322

A.M.N.H. 5, i, p. 137

A.M.N.H. 5, i, p. 376

Carter A.M.N.H. 5, xv, p. 400

A.M.N.H. 4, xvi, p. 198

A.M.N.H. t, xvi, p. 197

A.M.N.H. t, xvi, p. 197

P. Acad. Nat. Sci., Philad., 1884, p. 207 , var, ramotubulati Carter

P. Acad. Nit. Sci., Philad., 1884, p. 207

Reniera calyx A.M.N.H. $\quad$ t, xvi, p. 196 A.M.N.H. $5, \quad x, p .124$

" crassa
$" \quad$ crateriformis
" ligitata
fibulata

Carter A.M.N.H. t, xviii, p. 312

A.M.N.H. 5, $\quad$, p. 115

Jour. Linn. Soc, vol. xxi, p. 71

A.M.N.H. 5, ix, p. 287

P. Acad. Nat. Sci., Philad., 1884, p. 205

A.M.N.H. 4, xiv, p. 250

A.M.N.H. t, xvi, p. 196

A.M.N.H. 5, ix, p. 284 
Reniera fibulifera vasiformis

Rhaphidhistia spectabilis

\section{vermiculata}

Rhaphidotheca aftinis

A.M.N.H. 5, vi, p. 48 Carter A.M.N.H. 5, xviii, p. 445 A.II.N.H. 5, iii, p. 300 A.M.N.H. 5, ix, p. 354 A.M.N.H. 5, i, p. 140 J. R. Micro. SToc., vol. ii, p. 497 J. R. Hicro. Soc., vol. ii, p. 498 Rhaphiodesma floreum Rhaphiophora patera Rhaphyrus Griffithsii Rhizochalina carotta

Rinalda uberrima oleracea

Rossella antarctica

" philippinensis

, philippensis

, velata

Samus anonyma

„, anonymus

", complicatus

"parasiticus

"simplex

Sarcocornea nodosa

Sarcomella medusa

Sarcotragus fœtidus

Schmidtia clavatí

Semisuberites arctica

Spirastrella cunctatrix

A.M.N.H. 5, vi, p. 59

Carter A.M.N.H. 5, vi, p. 61

, A.M.N.H. 5, vi, p. 60

,$\quad$ A.M.N.H. 5, vi, p. 60

, A.M.N.H. 5, xv, p. 214

A.M.N.H. 5, viii, p. 251

A.M.N.H. 4, xvi, p. 193

A.MI.N.H. $\quad 4, \quad$ xvi, p. 196

Carter A.M.N.H. 4, $\mathrm{xx}_{\mathrm{p}}$ p. 39

A.M.N.H. 5, ix, p. 351

A.M.N.H. 5, xvii, p. 113

Jour. Linn. Soc., vol. xxi, p. 75 "var. porcata Carter A.M.N.H. 5, xvii, p. 115
". robusta Spongelia avara $\quad$ P. Acad. Nat. Sci., Philad., 188t, p. 203

$" \quad$ incrustans

" pallescens

, stellidermata

Spongia aculeata

" agaricina

" cancellata

, clavata
A.M.N.H. 4, xvi, p. 193

A.M.N.H. $\quad 4$, xviii, p. 232

Carter A.M.N.H. 5, xv, p. 219

A.M.N.H. 4, xvi, p. 194

A.M.N.H. 4, xvi, p. 194

A.M.N.H. 4, xvi, p. 194

A.M.N.H. 4, xvi, p. 196 
Spongia compressa

$\begin{array}{ll}" & \text { coriacea } \\ " & \text { Dysoni } \\ ", & \text { muricata } \\ \text { ofticinalis }\end{array}$

$$
\begin{gathered}
\not \quad \text { othahetica } \\
\text {, villosa } \\
\text { Spongilla alba }
\end{gathered}
$$

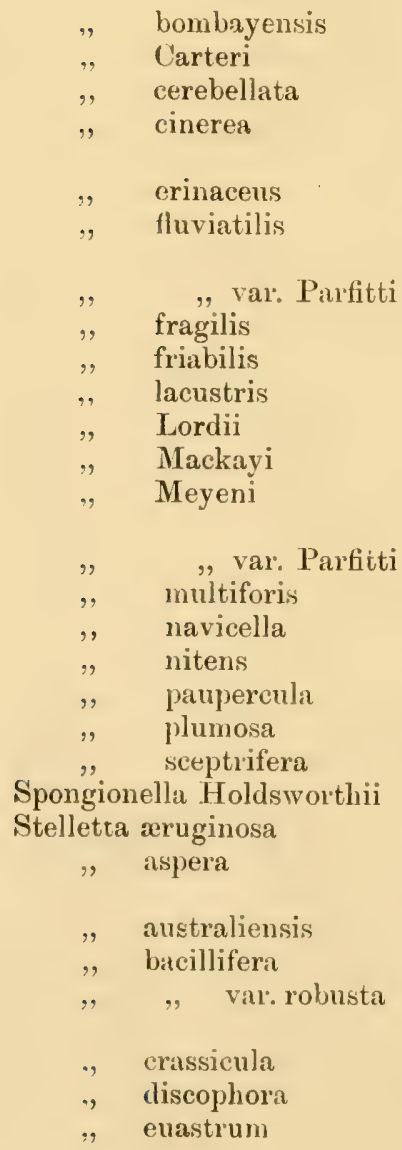

A.M.N.H. t, xvi, p. 194 A.N.N.H. 5, xiv, p. 17 A.M.N.H. 5, ix, p. 350 A.M.N.H. $\quad 4, \mathrm{xvi}, \mathrm{p} .194-5$ A.M.N.H. $\quad$ t, xvi, p. 192 A.M.N.H. t, xviii, p. 231 A.M.N.H. 5, ix, p. 272 Jour. Limm. Soc, vol. xxi, p. 63 A.M.N.H. t, xvi, p. 193 A.M.N.H. 4, xvi, p. 194 Carter A.M.N.H. 2, iv, p. 83 A.M.N.H. 2, $\mathrm{xx}, \mathrm{p} .21$ A.M.N.H. 5, vii, p. 88 A.M.N.H. 5, x, p. 369 A.M.N.H. 5, vii, p. 86 A.M.N.H. 5, vii, p. 88 Carter A.M.N.H. 2, iv, p. 82 A.M.N.H. 5, vii, p. 263 A.M.N.H. 5, vii, p. 91 A.M.N.H. 4, xvi, p. 199 A.M.N.H. 5, vii, p. 93 Carter A.M.N.H. 5, vii, p. 93 A.M.N.H. 5, $\mathrm{xv}, \mathrm{p} .18$ A.M.N.H. 2, iv, p. 83 A.M.N.H. 5, vii, p. 87 A.M.N.H. 5, vii, p. 89 Carter A.M.N.H. $5, \quad$ xv, p. 19 A.M.N.H. $\quad 2, \quad$ iv, p. 84 A.M.N.H. 5, vii, p. 93 A.M.N.H. $\quad 4, \quad$ i, p. 247 A.M.N.H. 5, vii, p. 88 A.M.N.H. 5, vii, p. 87 A.M.N.H. 5, vii, p. 89 A.MI.N.H. $5, \quad$ vii, p. 86 Carter A.MI.N.H. 2, iv, p. 8.5 A.M.N.H. 5, vii, p. 93 A.N.N.H. 4, xvi, p. 193 Carter A.M.N.H. 5, xvii, p. 123 A.M.N.H. 4, vii, p. 7 A.M.N.H. 4, xvi, p. 198 A.M.N.H. 5, xi, p. 350 "Jour. Limm. Soc., vol. xxi, p. 78 " A.M.N.H. 5. xi, p. 351 A.M.N.H. 5, xvii, p. 123 A.M.N.H. 5, vii, p, 371 A.M.N.H. $\quad 4, \quad$ xvi, p. 198 A.M.N.H. $\quad 4, \quad$ xvi, p. 198 
Stelletta geodides

$\begin{array}{ll}, & \text { globostellata } \\ , & \text { Grubii } \\ & \text { lactea }\end{array}$

,2 mammilliformis

, ochracea

, pachastrelloides

, reticulata

, tethyopsis

Stellettinopsis coriacea

$\begin{array}{ll}" & \text { corticata } \\ " & \text { lutea } \\ ", & \text { purpurea } \\ & \text { simplex }\end{array}$

tuberculata

Stelletites haldonensis

Stelospongus cribrocrusta

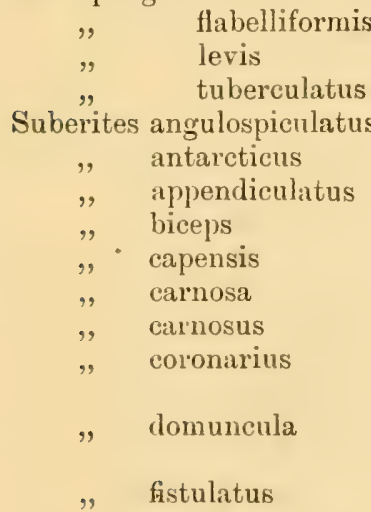

$\begin{array}{ll}, & \text { flabellatus } \\ , & \text { fuliginosus } \\ , \quad & \text { globosa } \\ , \quad \text { insignis } \\ , \quad \text { massa } \\ , \quad \text { montalbidus } \\ , \quad \text { montiniger }\end{array}$

\begin{tabular}{|c|c|c|}
\hline \multirow{4}{*}{$\begin{array}{c}\text { Carter } \\
,\end{array}$} & & 5 , \\
\hline & A.M.N.H. & 5, xvii, p. 12 \\
\hline & A.M.N.H. & xi, p. 35 \\
\hline & A.MI.N.H. & xvi, p. 19 \\
\hline \multirow[t]{2}{*}{ Carter } & A.M.N.H. & vii, p. \\
\hline & A.M.N. & xvi, p. 19 \\
\hline " & A.M.N. & xrii, p. 12 \\
\hline \multirow[t]{2}{*}{, } & L.N.H. & 5, xviii, p. \\
\hline & A.M.N.H. & 4, xviii, p. \\
\hline & A.M.N.H. & xi, p. \\
\hline ", & A.M.N.H. & vi, p. 13 \\
\hline \multirow[t]{2}{*}{ ", } & A.M.N.H. & 5, xvii, p. \\
\hline & A.M.N.H. & iii, $\mathrm{p}$. \\
\hline " & A.I & 5, xviii, p. \\
\hline ", & A.M.N & 5, xviii, p. \\
\hline \multirow[t]{2}{*}{, } & A. & iii, p. \\
\hline & A & 5, xvii, p. \\
\hline " & A. & 5, xvii, \\
\hline \multirow[t]{2}{*}{, } & A.1 & vii, p. \\
\hline & A.M.N.H. & 5, xviii, I \\
\hline \multirow[t]{2}{*}{$"$} & A. & $\mathrm{xv}, \mathrm{p}$. \\
\hline & A.MI.N & $x v, p$. \\
\hline \multirow[t]{2}{*}{ Carter } & A.M.N & $x v, p$. \\
\hline & A.M.N. & iii, 1 \\
\hline \multirow{2}{*}{3} & A.M.N & ix, \\
\hline & A.I & xvi, p. \\
\hline \multirow{2}{*}{ Carter } & A.I & xvii, p. \\
\hline & A.M.N.H. & ix, p. \\
\hline \multirow{2}{*}{ "Jou } & Soc., & xxi, p. \\
\hline & A & 5 \\
\hline Carter & A. & 5 , \\
\hline \multirow[t]{2}{*}{ Jour } & & xxi, p. 7 \\
\hline & A. & xvi, p. 19 \\
\hline & A. & ix, p. 35 \\
\hline \multirow[t]{3}{*}{ Carter } & A.M.N & vi, p. 53 \\
\hline & & vii, p. 370 \\
\hline & A. & ix, p. 354 \\
\hline & A. & xvii, p. 117 \\
\hline & A.M.N.H. & iii, p. 347 \\
\hline & A. & 5 \\
\hline & A.M.N & vii, 1) $11 \mathrm{c}$ \\
\hline & A.MI.N.H. & 5, xvii, p. 11 \\
\hline & A. & 4, xviii, p. \\
\hline & A.M.N & 5 , \\
\hline Carter & A. & 5 \\
\hline & A. & 5 , \\
\hline & A.M.N.H. & vi, P. 250 \\
\hline
\end{tabular}


Suberites parasitica

, spinispirulifer

,$\quad$ spinispirulifera

, stelligerus

" trincomaliensis

, vestigium

, Wilsoni

Sycandra Ramsayi

Sycon rhaphanus

Sycothamnus alcyoncellum

Sympagella nux

Taonura flabelliformis

Tedania digitata

, val. verrucosa

Teichonella labyrinthica

, prolifera

Terpios corulea

, fugax

Tethea muricata

'Tethya antaretica

, arabica

, atropurpurea

, casula

, Cliftoni

, cranium

" , var. abyssorum Carter

, ., " australiensis,

, ,", infrequens,

, , , robusta

, dactyloider

, lyncurium
A.M.N.H. 5, ix, p. 353

Carter A.M.N.H. 5, xvii, p. 119

A.M.N.H. 5, iii, p. 345

A.M.N.H. 5, xviii, p. 456

A.M.N.H. 5, x, p. 124

Jour. Linn. Soc., vol. xxi, p. 74

Carter A.M.N.H. 5, vi, p. 52

A.M.N.H. $\quad 5, \quad \mathrm{xv}$, p. 113

A.M.N.H. 5, xvii, p. 116

A.M.N.H. 5, xvii, p. 116

A.M.N.H. 5, xviii, p. 35

Midland Naturalist, vol. iii, p. 195

A.M.N.H. 5, xviii, p. 38

A.N.N.H. 4, xii, p. 361

A.M.N.H. 4, xii, p. 368

Carter A.M.N.H. 5, x, p. 108

A.M.N.H. 5, xvii, p. 52

Carter A.M.N.H. 5, xvii, p. 53

A.M.N.H. 5, ii, p. 37

A.M.N.H. 5, xviii, p. 38

A.M.N.H. 5, ii, p. 35

A.M.N.H. 5, xviii, p. 146

A.M.N.H. 5, ix, p. 355

A.M.N.H. 5, ix, p. 355

A.M.N.H. 5, ii, p. 174

Carter A.M.N.H. 4, ix, p. 412 Phil. Trans. Roy. Soc., vol. clx viui, p. 287

Carter A.M.N.H. 4, iv, p. 3

A.M.N.H. 4, xvi, p. 198

A.M.N.H. 4, vi, p. 176

A.M.N.II. 4, xvi, p. 198

A.M.N.H. 4, viii, p. 99

A.M.N.H. 4, xvi, p. 198

A.M.N.H. 5, xvii, p. 122

A.M.N.H. 4, xvi, p. 198

A.M.N.H. 4, xviii, p. 405

A.M.N.H. 5, xvii, p. 127

A.M.N.H. 4, xviii, p. 405

Jour. Linn. Soc., vol.xxi, p. 79

A.M.N.H. 4, iii, l. 15

A.M.N.H. 4, ix, p. 82

A.M.N.H. 4, xvi, p. 198

Jour. Linn. Soc., vol.xxi, p. 79

A.M.N.H. 4, iv, p. 7

A.M.N.H. 4, xvi, p. 198

P. Acad. Nat. Sci., Philad., 1884, p. 208 
Tethya merguiensis

$$
\begin{array}{ll}
, & \text { sphærica } \\
, & \text { stipitata } \\
, & \text { zetlandica }
\end{array}
$$

Tetilla polyura

Textiliforma foliata

Thalysias massalis

$$
\text { " subtriangularis }
$$

tener

Thecophora semisuberites

Thenea fenestrata

$$
\text { , muricata }
$$

,Wallichii

Theonella Swinhoei

Thoosa socialis

Tisiphonia annulata

" nana penetrians

$$
\begin{array}{ll}
", & \text { globosa } \\
, & \text {, var. rugosa } \\
", & \text { horrida } \\
, & \text { pernucleata }
\end{array}
$$

Trachyclidus levispirulifer

Trichogypsia villosa Trichostemma hemisphericum Trikentrion leve

$$
\begin{gathered}
\text { Tuba acapulcaensis } \\
, \quad \text { armigera } \\
, \text { digitalis } \\
., \quad \text { Eschrichtii } \\
, \quad \text { lineata } \\
, \quad \text { longissima } \\
, \quad \text { plicifera } \\
\text {, poculum } \\
, \text { sororia }
\end{gathered}
$$$$
\text { muricatum }
$$

Phil. Trans. Roy. Soc., vol. clxviii, p. 287

Carter A.M.N.H. 5, xi, p. 366 Jour. Linn. Soc., vol. xxi, p. 80 A.M.N.H. 5, ix, p. 860

Carter A.M.N.H. 5, xviii, p. 460 A.M.N.H. 4, ix, p. 417 A.M.N.H. $\quad 4, \quad x v i$, p. 198 Carter A.M N.H. 5, xvi, p. 288 A.M.N.H. 5, xvii, p. 50 A.M.N H. 4, xvi, p. 196 Carter Jour. Linn. Soc., vol.xxi, p. 70 A.M.N.H. t, xvi, p. 198 A.M.N.H. 5, $\quad \mathrm{xı}$, p. 362 A.M.N.H. 5, xi, p. 362 A.M.N.H. 5, xi, p. 362 A.M.N.H. 4, xii, p. 438 A.M.N.H. 4, xii, p. 441 Carter A.M.N.H. 5, vi, p. 56 A.M.N.H. 5, vii, p. 370 A.M.N.H. 5, vi, p. 140 A.M.N.H. 5, vi, p. 138 A.M.N.H. 5, vi, p. 141 A.M.N.H. 5, ix, p. 357 A.M.N.H. 5, xvii, p. 121 A.M.N.H. 5, xviii, p. 457 A.M.N.H. 5, xviii, p. 457 A.M.N.H. 4, vi, p. 178 A.M.N.H. t, xvi, p. 198 A.M.N.H. 5, iii, p. 343 A.M.N.H. 5, xvi, p. 357 A.M.N.H. t, viii, p. 1 "Nidland Nitturalist, vol. iii, p. 195 Carter A.M.N.H. 5, iii, p. 294 A.M.N.H. 5, $\quad x$, p. 114 A.M.N.H. 5, iii, p. 293 Carter A.M.N.H. 5, ix, p. 279 A.M.N.H. 4, xvi, p. 194 A.M.N.H. 5, ix, p. 20.s A.M.N.H. $\quad t, \quad x v i$, p. $19 t$ A.M.N.H. 5, ix, p. 277 A.M.N.H. 5, ix, p. 365 A.M.N.H. 5, ix, p. 2i A.M.N.H. $\quad$ t, xvi, p. 194 A.M.N.H. t, xvi, p. 194 A.M.N.H, 5, ix, p. 365. Carter A.M.N.H. 5, xw, p. 403 A.M.N.H. t, xvi, p. 194 
'Tuba tortolensis

'Tubella paulula

„ recurvati

" reticulata

, spinatir

Tubulodigitus communis

Uruguaya corallioides

Ute capillosa

Vioa Carteri

„Johnstonii

, Schmidtii

Verticillites anastomans

, helvetica

Wilsonellat austra'iensis

echinonematissima ",

Xenospongia patellifornis
P. Acad. Nat. Sci., Pliilad., 1884, p. 204 A.M.N.H. 4, xvi, p. 194 A.M.N.H. 5, vii, p. 96 A.M.N.H. 5, vii, p. 98 A.M.N.H. 5, vii, p. 97 Carter A.M.N.H. 5, vii, p. 96 A.M.N.H. 5, vii, p. 367

A.MI.N.H. 5, vii, p. 100 Phil. Trans. Roy. Soc., vol. clxviii, p. 288

A.M.N.H. 5, ix, p. 354

A.M.N.H. $\quad 4, \quad$ xvi, p. 197

A.M.N.H. 5, ix, p. 354

A.M.N.H. 5, xviii, p. 458

A.M.N.H. 5, ix, p. 354

A.M.N.H. 5, xiv, p. 27

A.M.N.H. 5, xiv, p. 27

Carter A.M.N.H. 5, xri, p. 366

A.M.N.H. 5, xix, p. 210

A.M.N.H. $\quad 4, \quad x v i$, p. 198

A.M.N.H. 5, ix, p. 357

Explanation of Abbremations.

A.M.N.H.-Annals and Magazine of Natural History.

J.K. Micro. Soc. - Journal of the Royal Microscopical Society. Jour. Linn. Soc.--Journal of the Linneun Society of LondonZoology.

P. Acarl. Nat. Sci., Philad.-Proceedings of the Acadeny of Natural Sciences of Philadelphia.

Phil. Trans. Roy. Soc.-Philosophical Transactions of the Royal Society of London. 
MELBOURSE: :

STILLWELI AND CO, PIINTERT, r95 (78) Coletiss sT. (EAsT). 






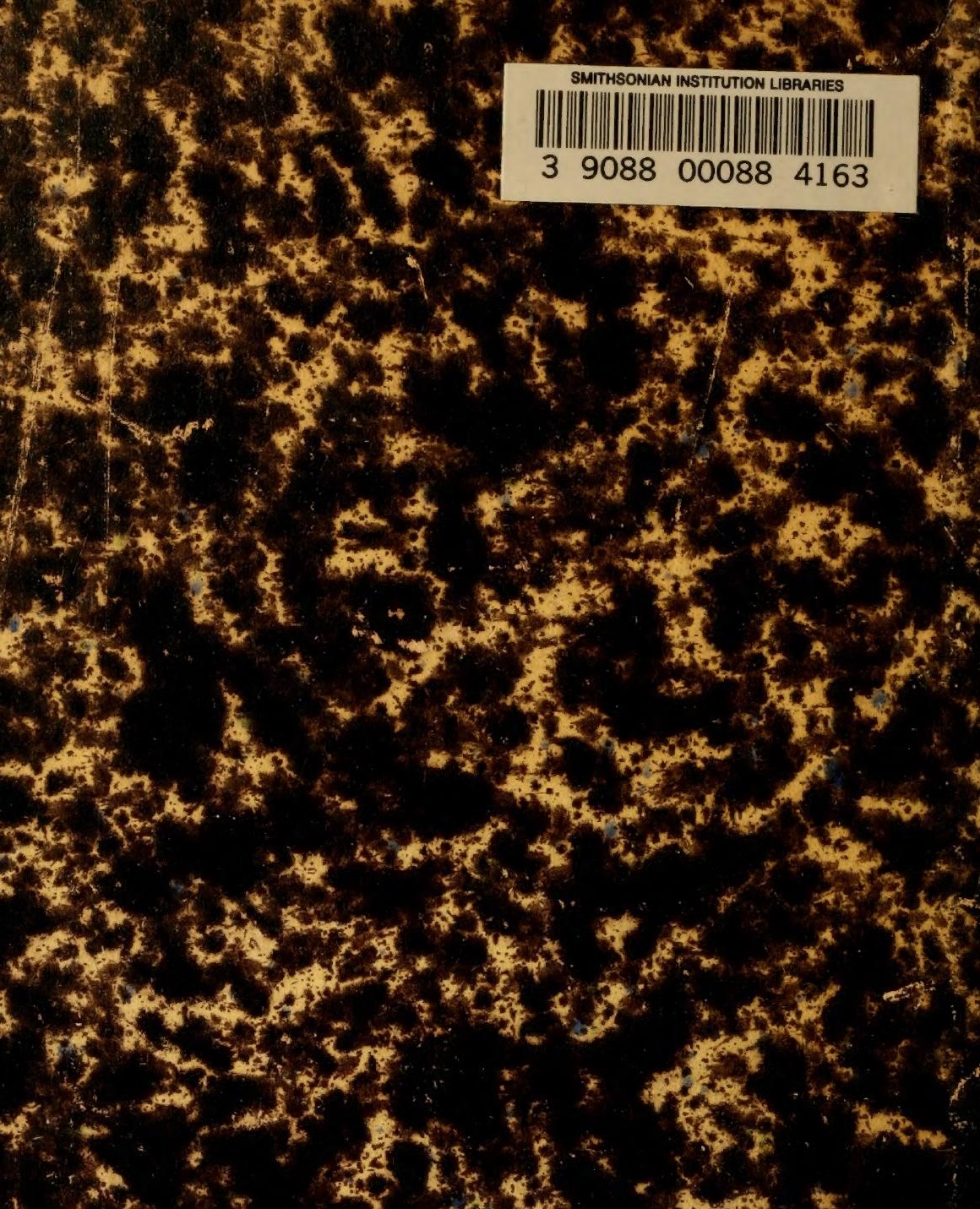

